which is seen to agree with (18), except for the fact that the coefficient of $\tau-\tau_{0}$ in the exponent of $e$ in (20) is the reciprocal of that in (19). This brings out the relation between systems (19) and (17). This is readily generalized to all systems like (17) which lead to self-adjoint differential equations of the second order with boundary conditions.

Harvard UNIVERSity, October 21, 1919.

\title{
ON A PENCIL OF NODAL CUBICS.
}

BY PROFESSOR NATHAN ALTSHILLER-COURT.

(Read before the American Mathematical Society December 31, 1919.)

Consider a pencil $\Gamma$ of nodal cubics having in common three collinear points, the double point, and the two tangents at this point.

1. Let $\Gamma_{n}$ be one of the cubics of the pencil $\Gamma$. A variable secant passing through one of the three basic collinear points $A, B, C$, say $A$, cuts $\Gamma_{n}$ in pairs of points which are projected from the double point $O$ by an involution of rays, the two tangents $O T_{1}, O T_{2}\left(T_{1}, T_{2}\right.$ are points of the basic line $\left.A B C\right)$ to $\Gamma_{n}$ at $O$ being a pair of conjugate elements in this involution. $^{1}$ The lines $O B, O C$, are obviously another pair of conjugate elements in this involution. The double lines $O M_{n}$, $O M_{n}{ }^{\prime}$, of this involution project from $O$ the two points of contact $M_{n}, M_{n}^{\prime}$ of the two tangents from $A$ to $\Gamma_{n}$.

When $\Gamma_{n}$ describes the pencil $\Gamma$ the two pairs of lines $O T_{1}$, $O T_{2}$ and $O B, O C$, remain fixed, by hypothesis, hence the involution

$$
O\left(T_{1} T_{2}, B C, M_{n} M_{n}, M_{n}{ }^{\prime} M_{n}{ }^{\prime}\right)
$$

is fixed, and therefore also its doubles lines $O M_{n}, O M_{n}^{\prime}$. Consequently: The points of contact of the pairs of tangents from one of the basic points to curves of the pencil $\Gamma$ lie on two fixed lines passing through the double point.

2. The corresponding point $A_{n}^{\prime}$ of $A$ on $\Gamma_{n}$ is projected

${ }^{1}$ M. Chasles, "Mémoire sur la construction des racines des équations du troisième et du quatrième degré," Comptes Rendus de l'Académie des Sciences, tome 41, p. 677. E. de Jonquières, Mélanges de Géométrie pure, 1856, p. 180. 
from $O$ by a line $O A_{n}{ }^{\prime}$ which is the harmonic conjugate of $O A$ with respect to $O T_{1}, O T_{2}{ }^{2}$

When $\Gamma_{n}$ varies the three lines $O A, O T_{1}, O T_{2}$ remain fixed, by hypothesis, therefore: The corresponding points on the

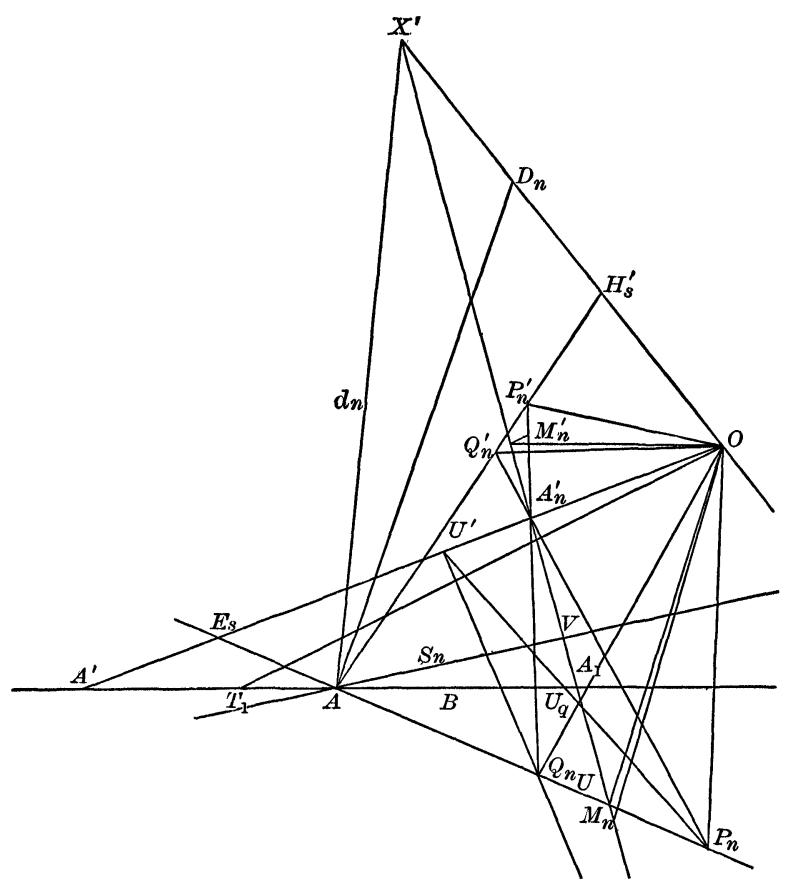

cubics of the pencil $\Gamma$ of any one of the basic points lie on a straight line passing through the double point.

3. The chord of contact $M_{n} M_{n}{ }^{\prime}$ of the two tangents $A M_{n}$, $A M_{n}{ }^{\prime}$ from $A$ to $\Gamma_{n}$ (1) passes through the corresponding point $A_{n}{ }^{\prime}(2)$ of $A$ on $\Gamma_{n}{ }^{3}$ The two lines $O A_{n}{ }^{\prime}, A_{n}{ }^{\prime} M_{n} M_{n}^{\prime}$ meet the line $A B C$ in two points $A^{\prime}, A_{1}$ harmonically separated by $B, C$, according to a theorem of McLaurin. ${ }^{4}$

When $\Gamma_{n}$ varies the point $A^{\prime}$ remains fixed (2), and since $B, C$ are also fixed, hence $A_{1}$ is a fixed point. Thus: The chords of contact of the pairs of tangents from one of the basic

${ }^{2}$ See, for instance, W. Binder, Theorie der unicursalen Plancurven. p. 293.

${ }^{3}$ de Jonquières, loc. cit., p. 226, prop. VII.

${ }^{4}$ de Jonquières, loc. cit., p. 237 , prop. XIV. 
points to the cubics of the pencil $\Gamma$ pass through a fixed point of the basic line.

4. Let $D_{n}$ be the tangential of $A$ on $\Gamma_{n}$, i.e., the point where $\Gamma_{n}$ is met again by the tangent to this cubic at the point $A$. In the involution (I) (1) to the line $O D_{n}$ corresponds the line $O A$. Since neither this involution, nor the line $O A$ vary with $\Gamma_{n}$, the line $O D_{n}$ is therefore fixed. Consequently: The tangentials of any of the basic points on the cubics of the pencil $\Gamma$ lie on a straight line passing through the double point.

5. Let $P_{n}$ be any point on $\Gamma_{n}$. The line $A P_{n}$ meets $\Gamma_{n}$ again in $Q_{n}$, and the two lines $O P_{n}, O Q_{n}$ are conjugate in the involution of rays (I) (1).

When $P_{n}$ varies with $\Gamma_{n}$ on the line $O P_{n}$, the line $O Q_{n}$, being the conjugate of the fixed line $O P_{n}$ in (I), will remain fixed. Hence: The lines projecting from a basic point the range of points determined by the pencil $\Gamma$ on any line passing through the double point, meet the respective cubics again on a fixed straight line passing through the double point.

$5 a$. The corresponding points of $A$ describe a straight line (2), hence (5): The lines joining a basic point to its corresponding points on the cubics of the pencil $\Gamma$ meet the respective curves again on a straight line passing through the double point.

6. The pairs of points determined on $\Gamma_{n}$ by a variable secant passing through $P_{n}$ are projected from $O$ by an involution of rays in which $O T_{1}, O T_{2}$ are a couple of conjugate elements, and the lines $O A, O Q_{n}(5)$ are another. ${ }^{1}$ In this involution to the ray $O P_{n}$ corresponds the line $O L_{n}$ projecting from $O$ the tangential $L_{n}$ of $P_{n}$ on $\Gamma_{n}{ }^{5}$

If the line $O P_{n}$ is maintained fixed while $\Gamma_{n}$ describes the pencil $\Gamma$, the point $Q_{n}$ will describe the range of points $O Q_{n}(5)$. Thus when $\Gamma_{n}$ varies the involution of rays just considered has besides the fixed couple $O T_{1}, O T_{2}$ also the fixed couple $O A, O Q_{n}$, hence this involution is fixed, and the conjugate $O L_{n}$ in this involution of the fixed ray $O P_{n}$ is also fixed. Consequently: The tangentials of the range of points determined by the curves of the pencil $\Gamma$ on a line passing through the double point, lie on a straight line also passing through the double point.

$6 a$. Since $L_{n}$ describes a line passing through $O$, its tangentials, which are the second tangentials of $P_{n}$, will also describe a straight line. In turn we may consider the tan-

${ }^{5}$ Binder, loc. cit. 
gentials of these tangentials, etc. Thus: The tangentials of any given order of the range of points determined by the pencil $\Gamma$ on a line passing through the double point, lie on a straight line also passing through the double point.

$6 b$. We have seen that the tangentials of $A$ describe a straight line (4), hence (6a): The tangentials of any given order of a basic point on the cubics of the pencil $\Gamma$ lie on a straight line passing through the double point.

7. The double elements $O T_{n}, O T_{n}{ }^{\prime}$ of the involution of rays $O\left(T_{1} T_{2}, A Q_{n}\right)(6)$ project from $O$ the points of contact $T_{n}, T_{n}{ }^{\prime}$ of the tangents $P_{n} T_{n}, P_{n} T_{n}^{\prime}$ from $P_{n}$ to $\Gamma_{n}$. Since this involution remains fixed when $P_{n}$ varies with $\Gamma$ on the line $O P_{n}$ (6), we have: The points of contact of the pairs of tangents drawn to the cubics of the pencil $\Gamma$ from the points which these curves respectively determine on a line passing through the double point, lie on two straight lines passing through the double point.

8. The lines $O A_{n}{ }^{\prime}, A_{n}{ }^{\prime} A_{1}$ (3) meet the secant $A P_{n} Q_{n}$ (6) in two points harmonically separated by $P_{n}$ and $Q_{n}{ }^{4}{ }^{4}$ Let $U \equiv\left(A P_{n} Q_{n}, A_{1} A_{n}{ }^{\prime}\right)$.

Let $R_{n}$ be any other point of $\Gamma_{n}$, and $S_{n}$ the third point of intersection of $\Gamma_{n}$ with $A R_{n}$. The lines $O A_{n}{ }^{\prime}, A_{n}{ }^{\prime} A_{1}(3)$ determine on the secant $A R_{n} S_{n}$ two points harmonically separated by $R_{n}, S_{n}{ }^{4} \quad$ Let $V \equiv\left(R_{n} S_{n}, A_{1} A_{n}{ }^{\prime}\right)$.

If the lines $O P_{n}, O R_{n}$ are kept fixed when $\Gamma_{n}$ describes the pencil $\Gamma$, the points $P_{n}, R_{n}$ will describe two ranges of points. The points $Q_{n}, S_{n}$ will describe two other ranges of points on the lines $O Q_{n}, O S_{n}(5)$. Hence the point $U$ will also describe a straight line, namely the harmonic conjugate of the fixed line $O A_{n}{ }^{\prime}(2)$ with respect to the couple of lines $O P_{n}, O Q_{n}$. Similarly $V$ will describe the harmonic conjugate of $O A_{n}{ }^{\prime}$ with respect to $O R_{n}, O S_{n}$. Thus we have

$$
\begin{aligned}
\left(P_{n} \cdots\right) \approx A\left(P_{n} \cdots\right) & \approx \\
& A_{1}(U \cdots) \\
& \approx(V \cdots) \approx A(V \cdots) \text { ॠ }\left(R_{n} \cdots\right) .
\end{aligned}
$$

It follows immediately from this construction that $(i)$ when $P_{n}$ coincides with $O$, the point $R_{n}$ likewise coincides with $O$, and (ii) when $P_{n}$ coincides with the point $\left(O P_{n}, A B C\right)$, the point $R_{n}$ coincides with $\left(O R_{n}, A B C\right)$. Hence: The lines joining the pairs of points determined by a variable cubic of the pencil $\Gamma$ on two fixed lines passing through the double point pass through a fixed point of the basic line. 
9. The tangent $P_{n} L_{n}$ to $\Gamma_{n}$ at $P_{n}$ joins two points which $\Gamma_{n}$ determines on the two lines $O P_{n}(5), O L_{n}(6)$. Consequently (8): The tangents to the curves of the pencil $\Gamma$ at their points of intersection with a line passing through the double point, pass through a fixed point of the basic line.

$9 a$. This proposition (9) may be applied to the range of points $A_{n}{ }^{\prime}$ (2) and also to the range of points $D_{n}(4)$. The reader may formulate the resulting theorems.

10. The tangents $P_{n} T_{n}, P_{n} T_{n}{ }^{\prime}$ from $P_{n}$ to $\Gamma_{n}$ have their points of contact $T_{n}, T_{n}^{\prime}$ on two fixed straight lines (7). Each of these two tangents passes through a fixed point of the basic line, when $\Gamma_{n}$ varies (9), hence we have, relatively to the variable point $P_{n}$ of the range $O P_{n}$ : The pairs of tangents drawn to the cubics of the pencil $\Gamma$ from the points which these curves respectively determine on a fixed line passing through the double point, pass through a pair of fixed points of the basic line.

11. The corresponding point $P_{n}^{\prime}$ of $P_{n}$ on $\Gamma_{n}$ lies on the line $O P_{n}{ }^{\prime}$ which is harmonically separated from $O P_{n}$ by the tangents $O T_{1}, O T_{2}$ to $\Gamma_{n}$ at $O,{ }^{2}$ therefore when $P_{n}$ varies with $\Gamma_{n}$ on $O P_{n}$, the line $O P_{n}{ }^{\prime}$ remains fixed. Thus: $A$ line through the double point meets the cubics of the pencil $\Gamma$ in a range of points the corresponding points of which on the respective curves lie on a straight line passing through the double point; and by virtue of (8): The lines joining the pairs of corresponding points thus obtained pass through a fixed point of the basic line.

12. Since the points of contact $T_{n}, T_{n}{ }^{\prime}$ of the tangents from the variable point $P_{n}$ of $O P_{n}$ to the variable cubic $\Gamma_{n}$ describe two straight lines (7), hence the variable line $T_{n} T_{n}^{\prime}$ meets $A B C$ in a fixed point (8). Thus: The chords of contact of the pairs of tangents drawn to the cubics of the pencil $\Gamma$ from the points which these curves respectively determine on a fixed line passing through the double point, meet the basic line in a fixed point.

13. The chord of contact of the two tangents from $A_{n}{ }^{\prime}(2)$ to $\Gamma_{n}$ passes through the conjugate $A$ of $A_{n}{ }^{\prime}$ on $\Gamma_{n}{ }^{3}$ and meets the line $A_{n}{ }^{\prime} M_{n} M_{n}^{\prime}{ }^{\prime}$ (3) in the harmonic conjugate $X^{\prime}$ of the point $\left(O A, M_{n} M_{n}{ }^{\prime}\right)$ with respect to the couple $M_{n}, M_{n}{ }^{\prime}{ }^{4}{ }^{4}$

The pairs of lines projecting from $A$ the couples of corresponding points on $\Gamma_{n}$ form an involution of rays, one of the double elements of which is the line $A O$ joining $A$ to the double point $O$. Since $M_{n}, M_{n}^{\prime}$ are a pair of corresponding points (3), the line $A X^{\prime}$ is the second double element of this involution of rays. 
The line $A X^{\prime}$ has thus a definite geometric meaning relative to the point $A$ on $\Gamma_{n}$. It is sometimes more convenient to speak of this line in connection with the point $A$ alone, without having to mention the corresponding point of $A$. Such a line as $A X^{\prime}$ shall be referred to as "the double line" through $A$ relative to $\Gamma_{n}$.

When $A_{n}{ }^{\prime}$ varies with $\Gamma_{n}$ (2) the harmonic pencil $O\left(M_{n} M_{n}^{\prime} A X^{\prime}\right)$ has three rays $O M_{n}, O M_{n}^{\prime}$ (1), $O A$, fixed, hence the fourth ray $O X^{\prime}$ is also fixed. Consequently: The double line, relative to a curve of the pencil $\Gamma$, through a basic point meets the chord of contact of the two tangents to this cubic from the same basic point, on a fixed line passing through the double point.

It may readily be shown that the line $O X^{\prime}$ is identical with the locus $O D_{n}$ of the tangentials of $A(4)$.

14. Let $K$ be the point of intersection of the line $P_{n} R_{n}(8)$ with $A B C$, and $W_{n}$ the third point of intersection of $P_{n} R_{n}$ with $\Gamma_{n}$. Let $P_{s}, R_{s}, W_{s}$ be the points of intersection of the lines $O P_{n}, O R_{n}, O W_{n}$ with any other cubic, say $\Gamma_{s}$, of the pencil $\Gamma$. The lines $P_{n} R_{n}, P_{s} R_{s}$ intersect in $K$, by virtue of prop. (8), i.e., $P_{s}, R_{s}, K$ are collinear.

Again, the lines $P_{n} W_{n}, P_{s} W_{s}$, intersect on $A B C$, by virtue of the same prop. (8). But $P_{n} W_{n} \equiv P_{n} R_{n}$ by construction, hence $P_{s} W_{s}$ passes through $K$, i.e., $P_{s}, W_{s}, K$ are collinear, consequently $P_{s}, R_{s}, W_{s}$ are collinear. Thus: (a) If three lines passing through the double point meet one of the cubics of the pencil $\Gamma$ in three collinear points, they meet every cubic of the pencil in three collinear points. (b) The bases of these triads of points concur on the basic line of the pencil.

15. The lines $P_{n} A, P_{n} A_{n}{ }^{\prime}$ projecting from the point $P_{n}$ (5) the couple of corresponding points $A, A_{n}^{\prime}(2)$ on $\Gamma_{n}$ meet $\Gamma_{n}$ again in a pair of corresponding points. ${ }^{6}$ The line $P_{n} A$ meets $\Gamma_{n}$ again in $Q_{n}(6)$, hence $P_{n} A_{n}{ }^{\prime}$ meets $\Gamma_{n}$ again in the corresponding point $Q_{n}{ }^{\prime}$ of $Q_{n}$ on $\Gamma_{n}$. Thus the corresponding points $P_{n}{ }^{\prime}, Q_{n}{ }^{\prime}$ of $P_{n}, Q_{n}$ on $\Gamma_{n}$ are collinear with $A$, and therefore the lines $O P_{n}{ }^{\prime}, O Q_{n}{ }^{\prime}$, are conjugate in the involution (I) (1).

The lines $A P_{n}, A P_{n}{ }^{\prime}$, projecting from $A$ the pair of corresponding points $P_{n}, P_{n}^{\prime}$ are harmonically separated by the double lines $A O, A X^{\prime}$ of the involution projecting from $A$ the pairs of corresponding points on $\Gamma_{n}(13)$. The line $O X^{\prime}$ cuts

${ }^{6}$ de Jonquières, loc. cit., p. 239, prop. XV. 
the harmonic pencil $A\left(O X^{\prime} P_{n} Q_{n}\right)$ in four harmonic points $O, X^{\prime}, H_{s}, H_{s}{ }^{\prime}$. The corresponding points $P_{n}{ }^{\prime}, Q_{n}{ }^{\prime}$ of the points $P_{n}, Q_{n}$ are thus the intersection of $A H_{s}{ }^{\prime}$ with the harmonic conjugates $O P_{n}{ }^{\prime}, O Q_{n}{ }^{\prime}$ of $O P_{n}, O Q_{n}$ with respect to $O T_{1}, O T_{2}$.

If the secant $s \equiv A P_{n} Q_{n}$ is maintained fixed, while $\Gamma_{n}$ varies, the pair of lines $O P_{n}, O Q_{n}$ will describe the involution (I) of which the pair of points $P_{n}, Q_{n}$ will describe the section by $s$. The lines $O P_{n}{ }^{\prime}, O Q_{n}{ }^{\prime}$ will describe the same involution (I): The line $O X^{\prime}$ will remain fixed (13), the points $X^{\prime}$ and $U(8)$ lying on the line $A_{1} A_{n}{ }^{\prime} M_{n} M_{n}{ }^{\prime}(2,3)$. Of the four harmonic points $O, X^{\prime}, H_{s}, H_{s}{ }^{\prime}$ the points $O, H_{s}$ are fixed, hence the two others $H_{s}{ }^{\prime}, X^{\prime}$ will describe on the fixed line $O X^{\prime}$ two superposed projective ranges the double elements of which are the points $O$ and $H_{s}$. Thus:

$$
\begin{array}{r}
O\left(P_{n}{ }^{\prime} Q_{n}{ }^{\prime} ; \cdots\right) \pi O\left(P_{n} Q_{n} ; \cdots\right) \text { ॠ }\left(P_{n} Q_{n} ; \cdots\right) \pi(U \cdots) \\
\text { ॠ } A_{1}(U \cdots) \text { त }\left(X^{\prime} \cdots\right) \pi\left(H_{s}^{\prime} \cdots\right) \text { ॠ } A\left(H_{s}^{\prime} \cdots\right) .
\end{array}
$$

This construction establishes a one-to-two projective correspondence between the pencil of lines $A\left(H_{s}{ }^{\prime} \cdots\right)$ and the pairs of lines of the involution (I). Consequently ${ }^{7}$ : The corresponding points, on the cubics of the pencil $\Gamma$, of the points determined by this pencil on a fixed line passing through a basic point, lie on a nodal cubic having the same double point as the given pencil and passing through the basic point considered.

When the lines $O P_{n}, O Q_{n}$ coincide with the couple $O B, O C$ of (I) the above construction shows that the harmonic conjugates of $O B, O C$ with respect to the couple $O T_{1}, O T_{2}$ are the two tangents to this cubic at its double point $O$.

16. The lines $O P_{n}, O Q_{n}(8)$ being harmonically separated by the double elements $O M_{n}, O M_{n}^{\prime}$ of (I), the points $U_{p} \equiv\left(O P_{n}, M_{n} M_{n}{ }^{\prime}\right), U_{q} \equiv\left(O Q_{n}, M_{n} M_{n}{ }^{\prime}\right)$ are harmonically separated by $M_{n}, M_{n}{ }^{\prime}$. On the other hand $M_{n}, M_{n}{ }^{\prime}$ being a couple of corresponding points on $\Gamma_{n}(1)$, the lines $P_{n} M_{n}$, $P_{n} M_{n}{ }^{\prime}$ are conjugate in the involution of lines projecting from $P_{n}$ the couples of corresponding points on $\Gamma_{n}$, and since one of the double elements of this involution of rays is $P_{n} O$, therefore the second double element passes necessarily through the point $U_{q}$. Thus $P_{n} U_{q}$ is the double line (13) of $P_{n}$ relative to $\Gamma_{n}$. Similarly the line $Q_{n} U_{p}$ is the double line relative to $\Gamma_{n}$ through $Q_{n}$.

\footnotetext{
${ }^{7}$ de Jonquières, loc. cit., pp. 170-171.
} 
The four points $P_{n}, Q_{n}, U_{p}, U_{q}$, determine a complete quadrangle the diagonal points of which are $O \equiv\left(P_{n} U_{p}, Q_{n} U_{q}\right)$, $U \equiv\left(P_{n} Q_{n}, \quad U_{p} U_{q} \equiv A_{1} A_{n}{ }^{\prime}\right), \quad U^{\prime} \equiv\left(P_{n} U_{q}, \quad Q_{n} U_{p}\right)$. Thus $O U^{\prime}$ is the harmonic conjugate of $O U$ with respect to $O P_{n}$, $O Q_{n}$, hence $O U^{\prime}$ is identical with $O A^{\prime}(8)$, i.e., $U^{\prime}$ lies on the fixed line $O A^{\prime}$. Let $E_{s} \equiv\left(O A^{\prime}, P_{n} Q_{n}\right)$. The harmonic pencil $U\left(E_{s} A_{n}{ }^{\prime} O U^{\prime}\right)$ determines on $O A^{\prime}$ the four harmonic points $E_{s}, A_{n}{ }^{\prime}, O, U^{\prime}$.

If the secant $s \equiv A P_{n} Q_{n}$ is kept fixed, while the cubic $\Gamma_{n}$ varies, the pair of lines $O P_{n}, O Q_{n}$ will describe the involution (I) of which the couple of points $P_{n}, Q_{n}$ describe the section by the fixed line $s$. Of the four harmonic points $E_{s}, A_{n}{ }^{\prime}, O, U^{\prime}$ the points $O, E_{s}$ remain fixed, hence $A_{n}{ }^{\prime}, U^{\prime}$ describe on the fixed line $O A^{\prime}$ two superposed projective ranges with the points $O$ and $E_{s}$ as the double elements of the projectivity. Thus:

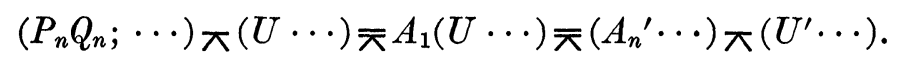

When $U^{\prime}$ coincides with $E_{s}$, the point $A_{n}{ }^{\prime}$ also coincides with $E_{s}$, hence $U$ will also coincide with $E_{s}$, consequently one point of the couple of elements of the involution $P_{n} Q_{n} \cdots$ coincides with $E_{s}$. Thus in the projective one-to-two correspondence established by $(a)$ between the points of the lines $O A^{\prime}$ and $s$ the point $E_{s}$ is a united element, hence $U^{\prime} P_{n}, U^{\prime} Q_{n}$ envelop a conic tangent to $s$, the point of contact being the trace on $s$ of the conjugate of $O A^{\prime}$ in (I), i.e., the line projecting from $O$ the third point of intersection of $\Gamma_{n}$ with $A A_{n}{ }^{\prime}$. Thus: The double lines of the points determined by the cubics of the pencil $\Gamma$ on a line passing through a basic point, envelop a conic tangent to the line considered.

When the lines $O P_{n}, O Q_{n}$ coincide with the lines $O B, O C$, the point $A_{n}{ }^{\prime}$ and hence the point $U^{\prime}$ will coincide with $O$, consequently: The conic is tangent to the lines projecting, from the double point, the other two basic points of $\Gamma$.

17. The points in which a line $l$ through the double point $O$ meets the cubics of the pencil $\Gamma$ have their tangentials on another line passing through $O(6)$. Hence if $l$ meets one of the cubics of $\Gamma$ in a point which coincides with its tangential, the same is true about the intersection of $l$ with any other cubic of $\Gamma$. Thus: If a line through the double point meets one of the cubics of the pencil $\Gamma$ in a point of inflexion, it meets all the cubics of the pencil in inflexional points. 
18. Let $I_{n}{ }^{\prime}, I_{n}{ }^{\prime \prime}, I_{n}{ }^{\prime \prime \prime}$ be the three points of inflection of $\Gamma_{n}$. The lines $O I_{n}{ }^{\prime}, O I_{n}{ }^{\prime \prime}, O I_{n}{ }^{\prime \prime \prime}$ meet every cubic of the pencil $\Gamma$ in three collinear points (14a), and all these points are points of inflection (17). Consequently: The points of inflection of all the cubics of the pencil $\Gamma$ lie on three straight lines concurring in the double point.

19. From (18) and (14b) it follows: The inflectional lines of the cubics of the pencil $\Gamma$ form a pencil having its vertex on the basic line.

It may be added that this vertex is the harmonic conjugate, with respect to the couple $A, A_{1}$, of the trace on $A B C$ of the locus of the tangentials of $A(4)$.

UNIVERSITY OF OKLAHOMA, October, 1919.

\section{DEFINITION AND ILLUSTRATIONS OF NEW ARITHMETICAL GROUP INVARIANTS.}

BY PROFESSOR E. T. BELL.

1. Arithmetical instances of groups are still sufficiently uncommon to make any new occurrence a matter of interest. Many significant group concepts have, of course, been implicit in arithmetic since at least the times of Euler and Gauss, notably in the theories of power residues, the automorphics of binary quadratic forms, and principal genera. More recently Miller has directly applied groups to quadratic residues and other topics.

This note contains the definition and a few illustrations, shorn of algebraic and other details, of certain group invariant relations for arbitrary integers, which are believed to be fundamentally distinct from previous group phenomena in arithmetic. These relations are genuinely arithmetical in that they concern only integers, and they may legitimately be called group relations because they exist only in reference to groups.

The object of this note is merely to call attention to these invariants by exhibiting a few of the simplest; and as several preliminary definitions are required - the subject being newdevelopments and less obvious examples may be left to another 